\title{
A casse report: Bilateral buccally impacted maxillary permanent canines
}

Alam MM ${ }^{1}$ BDS, FCPS and Hossain MZ2 BDS, PhD

\begin{abstract}
:
A 13 Years old girl with bilateral buccally impacted maxillary canine, shows spacing in upper arch and esthetically unpleasent. Permanent canines are considered the foundation of esthetic smile and functional occlusion. ${ }^{1}$ Management of impacted maxillary canine is important because of its contribution to the esthetics and its role in establishing arch form. So, treatment protocol with managemen of the impacted maxillary canine cases is vital for the orthodontist and general dental surgeons.
\end{abstract}

Key wards : Impacted, esthetic smile and functional occlusion (Ban J Orthod and Dentofac Orthop, Oct 2011; Vol-2, No. 1, p 28-29)

\section{INTRODUCTON}

The maxillary canine is second only to the mandibular third molar in its frequency of impaction. ${ }^{2-7}$ The prevalence is about $1.5 \%$ and the canine becomes ectopic more often palatally than buccally with over double the frequency. ${ }^{8}$ In our country, incidence of canine impaction involving approximately $2.8 \%$ of the patients seeking orthodontic treatment. More recently, the work of Oliver et al (1989) has indirectly indicated that Asians may suffer from buccally impacted canines more frequently than from palatal canines. ${ }^{9}$ Mismanagement and failures in diagnosis may be costly in terms of clinical time both for the practioner and patient and in litigation if damage occurs to adjacent teeth and proceeds unchecked.Surgical exposure with orthodontic traction is more suitable for management of impacted canine cases. The majority of normally erupting maxillary canines should be palpable in the buccal sulcus by ten to eleven years of age. Those maxillary canines erupting after approximately 12.3 years in girls and 13.1 years in boys may be considered late. ${ }^{10}$

\section{DIAGNOSIS}

A 13 years old girl came to Dhaka Dental College and Hospital with the complaint of spacing in anterior segment of upper arch and unpleasent esthetic looking and smile. On examination, facial profile was straight, upper both permanent canines were absent, spacing present in upper anterior segment, edge to edge incisor relationship, molar relationship is Class 1. No palpable bulging present on buccal or palatal side of the alveolar ridge. Absence of distal tipping or migration of the lateral incisor. Peg, Conical or abnormal shape lateral incisor or congenitally missing other teeth were absent.

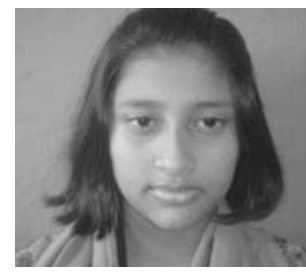

Extraoral- Frontal View

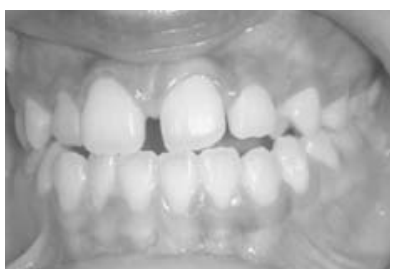

Intraoral Fontal View(Pretreatment)

\section{RADIOGRAPHIC EXAMINATION}

The patient with an ectopic maxillary canine must undergo a comprehensive assessment of the malocclusion including accurate localization of the ectopic canine. For accurate localization various radiographic examination should be carried out like peri apical radiograph, occlusal radiograph, panoramic radiograph, intraoral parallax radiograph etc.

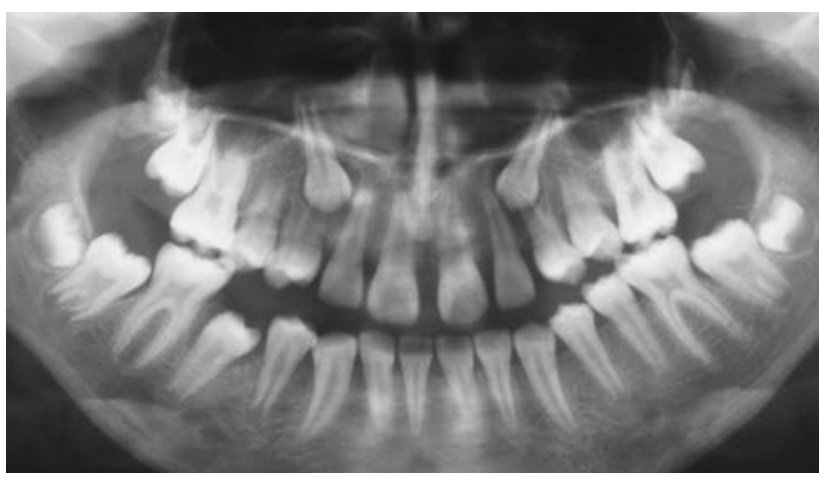

OPG

In panoramic radiograph shows bilateral maxillary canine impaction. Intraoral periapical radiographs with tube shift technique revealed that impacted canines were on buccal side.

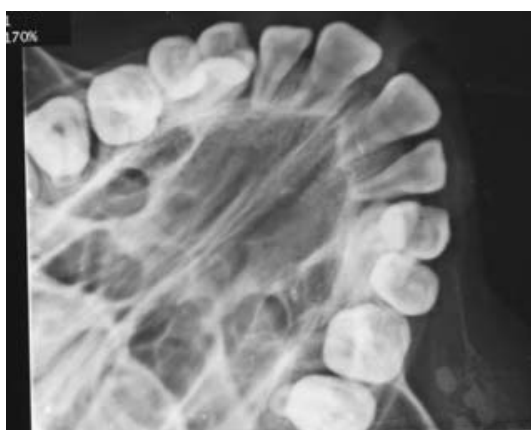

Occlusal Radiograph 


\section{AIM OF TREATMENT}

1. To insist eruption of upper impacted canine.

2. Proper alignment of upper anterior segment.

3. Establishment of proper overjet and overbite.

\section{TREATMENT PLAN}

1. Leveling and alignment with standard edgewise appliance.

2. Bilateral opening of space with open coil spring in between lateral incisor and first premolar.

3. Surgical exposure of impacted maxillary canines.

4. Orthodontic traction to insist eruption and proper alignment.

5. Proper interdigitation by use of class III and up -down elastics.

\section{SURGICAL PROCEDURE}

Under local anaesthesia a buccal flap was raised in the maxilla from canine to canine region. The crown of the canine was exposed and brackets were bonded to an accessible site. Ligature wire ( 0.009 inch s.s) passed through the bracket and brought out of the flap. Flap was then closed with sutures.

\section{TREATMENT PROGRESSION}

Impacted canines were guided towards normal alignment. To assess the progresss periodic intraoral periapical radiographs were taken. As both upper canines reached its normal level in the arch, leveling and alignment of lower arch and proper interdigitation was done by Class III elastics and up-down elastics.

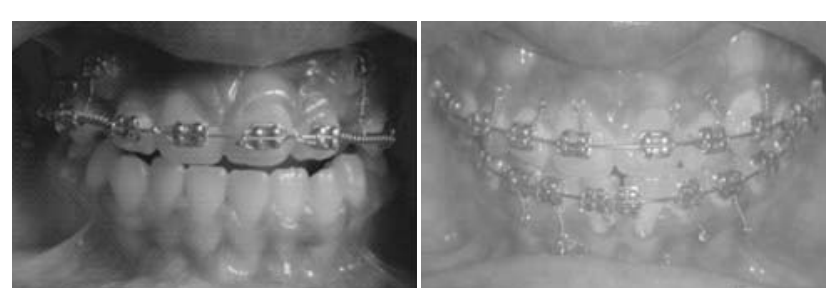

Intraoral Photograhps (Mid-treatment)

Post Treatment Photographs
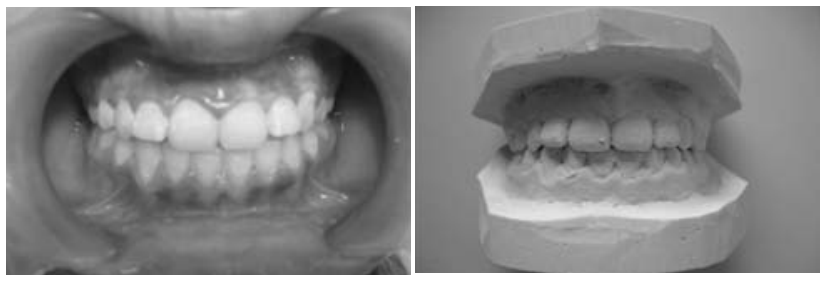

Intraoral Frontal View

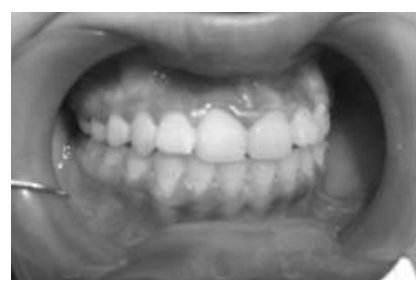

Right lateral View
Frontal View (Model)

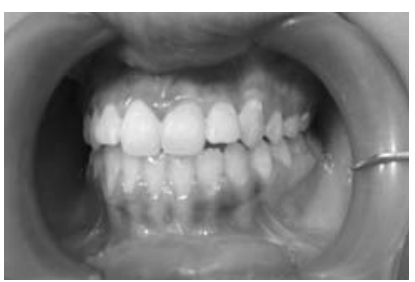

Left lateral View

\section{DISCUSSION}

Total treatment time was about 16 months. With the treatment result patient and her parents were happy with her improved appearance and function. Proper treatment in right time and co-operation of the patient are important for the success of any treatment.

\section{REFERENCES:}

1. Patrick F.Mcsherry. The ectopic maxillary canine; A review.Br.J.Orthod 1998;25:209-216.

2. Shapira Y,Kuftinec MM .Early diagnosis and interception of potential maxillary canine impaction.J Am Den Assoc .1998;129:14501454.

3. Sambataro S BaccettiT, Franchi L,Antonini F .Early predictive variables for upper canine impaction as derived from posteroanterior cephalograms .Angle Orthod .2004; 75:28-34.

4. Coulter J,Richerdson A. Normal eruption of the maxillary canine quantified in three -dimensions. Eur J Orthod .1997;18:444-456.

5. Langberg BJ, Peck S. Adequqcy of maxillary dental arch width in patients with palatally displaced canines . Am J Orthod . 2000; 118: 220-223.

6. Ericson S, Kurol J. Early treatment of palatally erupting maxillary canines by extraction of the primary canines. Eur $\mathrm{J}$ Orthod.1988;10:283-295.

7. Bishara SE. Clinical management of impacted canines .Semin Orthod1998;4 :87-98.

8. Ericson S,Kurol J .Radiographic examination of ectopically erupting maxillary canines. American Journal of orthodontics and Dentofacial orthopadeics .1987; 91:483-492.

9. Kuftinec M M, Strom D ,Shapira Y, The impacted maxillary canine : 1 .Review of concepts. ASDC J Dent Child.1995: 62 (5) :317 -324.

10. Hurme V. Ranges Of normality in the eruption of permanent teeth. Journal of Dentistry for Children 1949;16: 11-16.

\section{Corresponds to:}

\section{Dr. Md. Mahbub Alam}

BDS,FCPS (Orthodontics)

Lecturer, Department of Orthodontics.

Dhaka Dental College, Dhaka

Phone : +880-01711152983

E-mail : unique778@yahoo.com 